\title{
Observation of interspecies ion separation in inertial-confinement- fusion implosions
}

\author{
S. C. Hsu, ${ }^{1}$ T. R. Joshi, ${ }^{1}$ P. Hakel, ${ }^{1}$ E. L. Vold,${ }^{1}$ M. J. Schmitt, ${ }^{1}$ N. M. Hoffman, ${ }^{1}$ R. M. Rauenzahn, ${ }^{1}$
} G. Kagan ${ }^{1}$ X.-Z. Tang, ${ }^{1}$ R. C. Mancini, ${ }^{2}$ Y. Kim, ${ }^{1}$ and H. W. Herrmann ${ }^{1}$

Los Alamos National Laboratory, Los Alamos, New Mexico 87545, USA

Physics Department, University of Nevada, Reno, Nevada 8955\%, USA

PACS 52.57.-z- First pacs description

PACS 52.25.Fi - Second pacs description

PACS 52.70.La - Third pacs description

\begin{abstract}
We report direct experimental evidence of interspecies ion separation in direct-drive, inertial-confinement-fusion experiments on the OMEGA laser facility. These experiments, which used plastic capsules with $\mathrm{D}_{2} / \mathrm{Ar}$ gas fill (1\% Ar by atom), were designed specifically to reveal interspecies ion separation by exploiting the predicted, strong ion thermo-diffusion between ion species of large mass and charge difference. Via detailed analyses of imaging x-ray-spectroscopy data, we extract Ar-atom-fraction radial profiles at different times, and observe both enhancement and depletion compared to the initial 1\%-Ar gas fill. The experimental results are interpreted with radiation-hydrodynamic simulations that include recently implemented, first-principles models of interspecies ion diffusion. The experimentally inferred Ar-atom-fraction profiles agree reasonably, but not exactly, with calculated profiles associated with the incoming and rebounding first shock.
\end{abstract}

Interspecies ion separation has been proposed as a potential fusion-yield degradation mechanism in inertialconfinement-fusion (ICF) implosions [1] . Several ICF experimental campaigns [4] have reported yield or yield-ratio anomalies where interspecies ion separation within the hot spot is a possible explanation. Meanwhile, first-principles analytic theories for multi-ion-species diffusion 1, 2, 12 16 have been developed, some of which have been implemented into ICF implosion codes [17, 18, enabling us to quantitatively assess the magnitude of interspecies ion separation and yield degradation in ICF implosions. In addition, ion-Fokker-Planck [19, 20] and particle-in-cell kinetic simulations [3,21,22] are being used to address this problem, and are particularly appropriate for more-kinetic scenarios, e.g., exploding pushers, hotspot formation in ignition capsules, hohlraum plasmas, or near steep gradients at shock fronts and material interfaces. We do not address the question here of whether interspecies ion separation substantially affects the yield in ignition-class implosions on the National Ignition Facility [23], but point out that this and related work will help establish a validated capability to answer the question in a quantitative manner.

The purpose of this work is to complement and expand upon prior ICF experimental campaigns that relied on yield or yield-ratio anomalies as evidence for species separation. Here, based on direct-drive ICF implosions on the OMEGA laser facility [24, we provide direct, spatially resolved experimental evidence of interspecies ion separation via detailed analyses of imaging x-ray-spectroscopy data, which are less sensitive to other potential causes of yield degradation in an ICF implosion. In this work: (1) we exploit recently developed analytic theory [14, 15] of interspecies ion diffusion to design an ICF implosion that maximizes interspecies ion diffusion, such that it is observable via x-ray diagnostics; (2) rather than focusing on separation between fuel species, e.g., $\mathrm{D} /{ }^{3} \mathrm{He}$ or $\mathrm{D} / \mathrm{T}$, we use D and a trace amount of Ar, where both the choice of Ar itself and its pre-fill concentration are chosen to maximize the expected interspecies diffusion coefficient; and (3) we aim for a more-collisional implosion so that interpretations using the recently formulated interspecies-iondiffusion theories are appropriate. The main results in this Letter are the first direct identification of interspecies ion separation in an ICF implosion based on the analyses of spatially resolved x-ray-spectroscopy data, observation of spatially non-uniform Ar-concentration enhancement and depletion (compared to the spatially uniform 1 atom\% $\mathrm{Ar}$ 

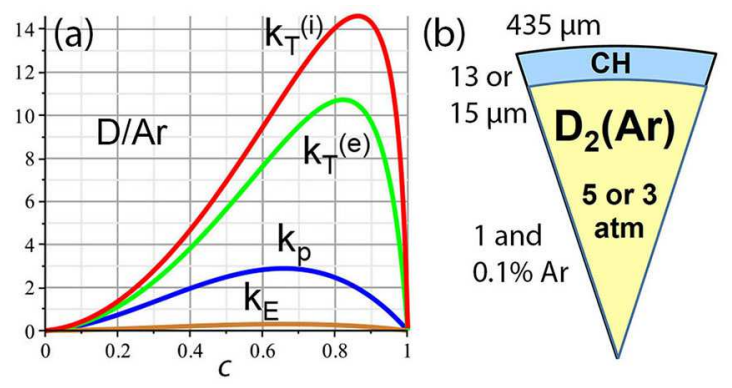

Fig. 1: (a) D/Ar interspecies diffusion ratios vs. $c \equiv \rho_{D} /\left(\rho_{D}+\right.$ $\left.\rho_{A r}\right)$ for ion thermo-diffusion $k_{T}^{(i)}$, electron thermo-diffusion $k_{T}^{(e)}$, baro-diffusion $k_{p}$, and electro-diffusion $k_{E}$, assuming charge state $Z_{A r}=18$. (b) We shot four target types: $15 \mu \mathrm{m} / 3 \mathrm{~atm}$ and $13 \mu \mathrm{m} / 5 \mathrm{~atm}$, with 1 or 0.1 atom\% Ar.

pre-fill) during the implosion, and reasonable agreement of the experimentally inferred Ar-atom-fraction profiles with radiation-hydrodynamic simulations that model interspecies ion diffusion from first principles.

We briefly discuss the theory that guided these experiments. The diffusive mass flux of the lighter ions relative to the center of mass in a plasma with two ion species can be written as [14, 15] $\vec{i}=-\rho D\left[\nabla c+k_{p} \nabla \log p_{i}+\right.$ $\left.\left(e k_{E} / T_{i}\right) \nabla \Phi+k_{T}^{(i)} \nabla \log T_{i}+k_{T}^{(e)} \nabla \log T_{e}\right]$, where $\rho$ is the total mass density, $D$ the classical diffusion coefficient, $c \equiv \rho_{l} / \rho$ the lighter-ion mass concentration, $p_{i}$ the ion pressure, $T_{i}$ and $T_{e}$ the ion and electron temperatures, respectively, $\Phi$ the electric potential, and $k_{p}, k_{E}, k_{T}^{(i)}, k_{T}^{(e)}$ the diffusion ratios for baro-diffusion, electro-diffusion, ion thermo-diffusion, and electron thermo-diffusion, respectively; $\vec{i}$ can also be written in terms of molarconcentration gradients [16]. Because the theory predicts larger diffusion ratios for ion species with a large mass and charge difference [15, we focused on a D/Ar mixture (rather than $\mathrm{D} /{ }^{3} \mathrm{He}$ or $\mathrm{D} / \mathrm{T}$ ). We chose Ar in particular because of its proven usefulness as an x-ray spectroscopic tracer in ICF implosions [25]31. We evaluated the diffusion ratios 15 for a D/Ar mixture versus $c$ [fig. [1(a)]; $k_{T}^{(i)}$ is the largest ratio and maximizes at $c=\rho_{D} /\left(\rho_{D}+\rho_{A r}\right) \approx 0.84$, corresponding to Ar number fraction $f_{A r} \equiv n_{A r} /\left(n_{A r}+n_{D}\right) \approx(1-c) /(1+19 c) \approx 1 \%$. Thus, we focused our attention on an implosion design with large $\nabla T_{i}$ and $f_{A r} \approx 1 \%$ to maximize ion thermodiffusion. Furthermore, we wanted relatively high $T_{i}$ and $D$ while keeping the Ar-D mean free path much smaller than the hot-spot size for most of the implosion. Although the theory 14 16] is not strictly correct within a shock front, it should nevertheless capture the qualitative, leading-order effects on interspecies ion separation during the passage and rebound of the first incoming shock.

We performed 1D HYDRA 32 simulations of directdrive OMEGA implosions with a 1-ns square pulse to arrive at the capsule designs in fig. 1(b). Table 1 summarizes the as-shot laser and target parameters and neutron- based measurements. To evaluate the amount of expected species separation, we also performed pre-shot 1D simulations using xRAGE [17] with its recently implemented two-ion-species transport model [16]. All our preshot xRAGE results showed relative deviations in $f_{A r}$ of $\geq 20 \%$, in some cases much larger, from the initial values $\left(f_{A r}=0.01\right.$ or 0.001$)$ over much of the implosion, giving us confidence that we could resolve the species separation based on error bars achieved in previous analyses of imaging x-ray-spectroscopy data.

The primary diagnostics are two x-ray multimonochromatic imagers (MMI) 33 with quasi-orthogonal views (mounted on TIMs 3 and 4, where TIM stands for a "ten-inch manipulator" diagnostic port), a streaked x-ray spectrometer (TIM 1), and a time- and space-integrated, absolutely calibrated x-ray spectrometer (TIM 2). Standard neutron diagnostics and full-aperture backscatter systems were also fielded. The MMIs used 10- $\mu$ m-diameter pinholes, setting the spatial resolution at $\gtrsim 10 \mu \mathrm{m}$, and recorded data on x-ray framing cameras between 3.3$5.5 \mathrm{keV}$ around the time of first-shock convergence. Each camera recorded four frames per shot at different times; frame trigger times are given in the figure captions. We obtained analyzable MMI data for several shots with initial $f_{A r} \approx 1 \%$ (table 1), and used the $\operatorname{Ar}-\mathrm{He} \beta$ (3.68 keV), Ly $\beta$ $(3.94 \mathrm{keV})$, and $\mathrm{Ly} \gamma(4.15 \mathrm{keV})$ lines in our spectroscopic analyses. Shots with initial $f_{A r} \approx 0.1 \%$, predicted to have much reduced ion thermo-diffusion, provided weaker spectral lines.

The key objective and result of this work is the experimental inference of $f_{A r}$ versus radius $r$. Deviation from the spatially uniform target-pre-fill value constitutes proof of interspecies ion separation. Figures 2 and 3 illustrate the key steps of the MMI data processing and analyses required to arrive at $f_{A r}(r)$. MMI data processing to obtain narrow-band images [fig. 2(b)] is based on the method of refs. 29, 31, and 34. Extraction of electron density $n_{e}$ and temperature $T_{e}$ versus $r$ are based on the emissivityanalysis method of ref. 28, Figure 2(a) shows one frame of MMI data after it has been converted from film density to intensity (arbitrary units). The photon-energy dependence of the MMI data is corrected using streaked and absolutely calibrated spectral data [35. Figure 2(b) shows the narrow-band images constructed from the data in fig. 2(a), and fig. 2(c) shows the space-integrated spectrum from fig. 2(a) before continuum subtraction.

The next step is to extract $n_{e}$ and $T_{e}$ radial profiles. Abel inversion is applied to each narrow-band image, e.g., fig. 2(b), giving argon emissivity (with continuum subtracted) versus $r$ for four radial zones of approximately $10-\mu \mathrm{m}$ width (set by both the size of MMI pinholes and spectral signal-to-noise considerations for each zone), at the time of the particular MMI frame. The time is approximately known based on the experimental trigger time of each frame of the x-ray framing camera, but due to cable delays and the finite time of the sweep across the camera's photocathode strips, we regard the quoted times as hav- 
Table 1: Summary of shots analyzed for this Letter. Yield (DD-n) and burn-weighted ion temperature $\left\langle T_{i}\right\rangle$ are from the 12-m neutron time-of-flight scintillator, and neutron bang times (BT) and burn widths (BW) are from the cryogenic neutron temporal diagnostic (cryoNTD). Note that BT corresponds to compression burn in these implosions.

$\begin{array}{lllllllll}\text { Shot \# } & \begin{array}{l}\text { laser } \\ \text { energy }(\mathrm{kJ})\end{array} & \begin{array}{l}\text { capsule OD }(\mu \mathrm{m}) / \\ \text { CH thickness }(\mu \mathrm{m})\end{array} & \begin{array}{l}\mathrm{D}_{2} / \text { Ar fill } \\ (\mathrm{atm})\end{array} & \begin{array}{l}\text { Ar } \\ (\text { atom\%) }\end{array} & \begin{array}{l}\text { yield } \\ (\text { DD-n })\end{array} & \begin{array}{l}\left\langle T_{i}\right\rangle \\ (\mathrm{keV})\end{array} & \begin{array}{l}\text { BT } \\ (\mathrm{ns})\end{array} & \begin{array}{l}\mathrm{BW} \\ (\mathrm{ps})\end{array} \\ 78197 & 21.2 & 863.2 / 13.1 & 5.0 & 1.01 & 1.18 \mathrm{E} 11 & 6.43 & 1.317 & 110 \\ 78199 & 21.1 & 861.8 / 13.1 & 5.0 & 1.01 & 1.35 \mathrm{E} 11 & 6.48 & 1.313 & 116 \\ 78201 & 26.2 & 874.5 / 14.8 & 3.18 & 1.12 & 8.06 \mathrm{E} 10 & 6.93 & 1.355 & 120\end{array}$

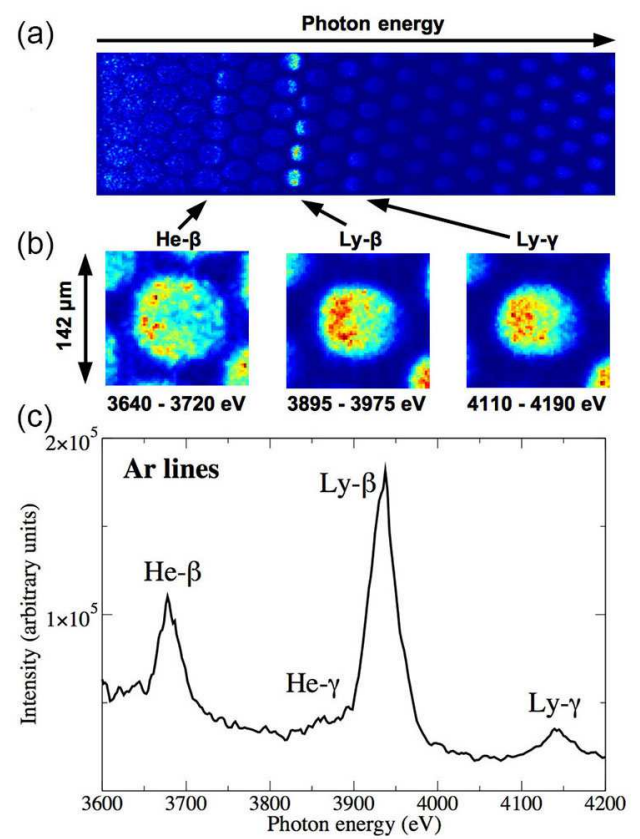

Fig. 2: (a) MMI data showing an array of spectrally resolved implosion-core pinhole images (intensity, arbitrary units) versus photon energy along the $x$ axis for shot 78199, TIM 3 , frame 2 (1.14-ns trigger time), (b) Ar $\mathrm{He} \beta, \mathrm{Ly} \beta$, and $\mathrm{Ly} \gamma$ narrowband images constructed from $80-\mathrm{eV}$-wide (as given below each image) vertical strips of the data in (a). (c) Space-integrated spectrum from (a) before continuum subtraction.

ing an uncertainty $\sim 50$ ps. The $\mathrm{Ar}-\mathrm{He} \beta$ and $\mathrm{Ly} \beta$ emissivity profiles are analyzed to provide $n_{e}$ and $T_{e}$ versus $r$ [fig. [3(a)] based on comparisons with detailed atomicphysics models. The underlying atomic database was generated via the fully relativistic path in the Los Alamos suite of codes RATS, ACE, and GIPPER [36, 37, followed by the collisional-radiative code ATOMIC 37 39. The resulting NLTE (non-local thermal equilibrium) spectral data were used in the intensity and emissivity analyses of the MMI data [figs. 3. 4(a), and 4(b)], as well as in the forward modeling of space-resolved spectra and narrowband images by the FESTR spectral post-processing code 40.41. Error bars for $T_{e}$ are obtained based on the greater of the $T_{e}$ resolution $(100 \mathrm{eV})$ in the atomic database mentioned above or the standard deviation of $T_{e}$ (e.g., average

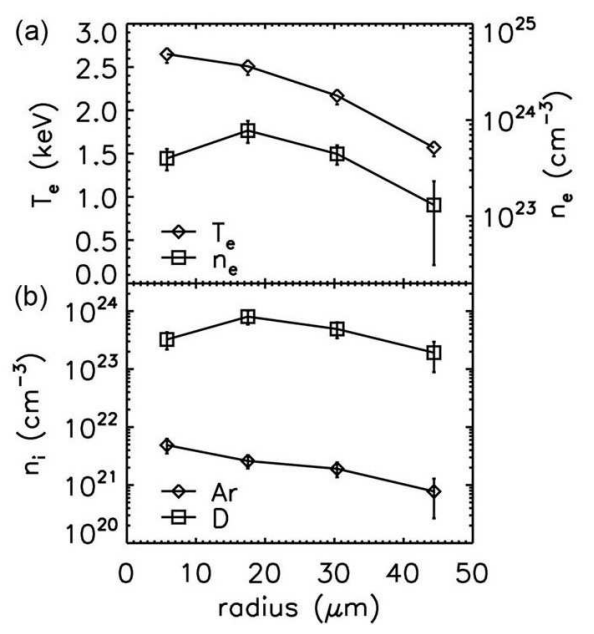

Fig. 3: (a) Electron temperature and density and (b) D and Ar ion densities versus radius, from analysis of MMI data (shot 78199, TIM 3, frame 2, 1.14-ns trigger time). See text for explanation of error bars.

of $63 \mathrm{eV}$ for the four radial zones for shot 78199, TIM 3, frame 2) as determined from the theoretical emissivity ratio of $\operatorname{Ly} \beta / \mathrm{He} \beta$ at four different values of $n_{e}\left(5 \times 10^{23}\right.$, $8 \times 10^{23}, 1 \times 10^{24}$, and $2 \times 10^{24} \mathrm{~cm}^{-3}$ ) in our regime of interest. Error bars for $n_{e}$ are obtained based on the greater of the uncertainties in the $T_{e}(100 \mathrm{eV})$ and $n_{e}$ resolutions $\left(1-2 \times 10^{23} \mathrm{~cm}^{-3}\right.$, depending on the density) in the database, or the standard deviation in $n_{e}$ (e.g., average of $1.1 \times 10^{23} \mathrm{~cm}^{-3}$ for the four radial zones for shot 78199 , TIM 3, frame 2) from assuming slightly higher and lower values of $n_{e}$ in the central zone (e.g., $4 \pm 1 \times 10^{23} \mathrm{~cm}^{-3}$ for shot 78199, TIM 3, frame 2), which is derived from the line broadening of each of the three Ar lines from the space-integrated spectrum.

The argon density $n_{A r}$ is determined using $n_{A r}=$ $n_{u} / F_{u}\left(T_{e}, n_{e}\right)$, where $n_{u}$ and $F_{u}$ are the upper-level number densities and fractional populations, respectively, of the particular line transition being used 42. $F_{u}\left(T_{e}, n_{e}\right)$ is retrieved from the atomic database based on the previously determined $n_{e}(r)$ and $T_{e}(r)$. Since the spectral lines are sufficiently optically thin [28], $n_{u}$ (arbitrary units) is extracted based on its proportionality to the corresponding observed line intensities, which are obtained from the space-resolved spectra extracted from annular regions of 
the implosion-core image by numerically integrating the area under the line after continuum subtraction. Finally, the known pre-fill amount of Ar closes the system of linear equations relating $n_{u}(r)$ to $n_{A r}(r)$ [42. Error bars for $n_{A r}$ are from propagation of uncertainties in $n_{u}$ (e.g., $9 \%$, $6 \%, 7 \%$, and $8 \%$ from the innermost to outermost radial zone for shot 78199 , TIM 3 , frame 2$)$ and $F_{u}\left(T_{e}, n_{e}\right)$ (e.g., $25 \%, 22 \%, 20 \%$, and $54 \%$ from the innermost to outermost radial zone for shot 78199 , TIM 3, frame 2). The deuterium density $n_{D}$ is inferred from quasi-neutrality $n_{e}=Z_{D} n_{D}+Z_{A r} n_{A r}$, and finally $f_{A r} \equiv n_{A r} /\left(n_{A r}+n_{D}\right)$. Error bars for $n_{D}$ and $f_{A r}$ use the propagated errors from $n_{e}$ and $n_{A r}$, and $n_{A r}$ and $n_{D}$, respectively. Figure 3 shows an example of $n_{e}, T_{e}, n_{A r}$, and $n_{D}$ versus $r$, and figs. 4 (a) and 4(b) show $f_{A r}(r)$ from different shots and MMI views and times. Results shown are the averages from the Ar$\operatorname{He} \beta, \operatorname{Ly} \beta$, and Ly $\gamma$ lines. The non-uniform spatial profiles of $f_{A r}$ and their deviation from the initial pre-fill value from several different shots and times constitute proof of interspecies ion separation between the D and Ar, which is the main result of this Letter.

To build confidence in our conclusions, we apply the same analysis procedure to synthetic $\operatorname{Ar}-\mathrm{He} \beta, \mathrm{Ly} \beta$, and Ly $\gamma$ images and space-resolved spectra. The synthetic data are generated using the FESTR code [41, using the data in fig. 3 and assuming $1 \mathrm{D}$ spherical symmetry, for two cases: (i) $n_{A r}$ as shown in fig. 3(b) and (ii) spatially uniform $f_{A r}=0.01$ with equal $n_{D}+n_{A r}$ as in (i). In both cases, we extract $f_{A r}(r)$ in good agreement with the $f_{A r}(r)$ in the synthetic data. This demonstrates that our analysis accurately extracts $f_{A r}(r)$ and is also able to distinguish between species separation versus no species separation.

We also note some limitations of our analysis. Firstly, our analysis assumes 1D spherical symmetry, introducing possible errors if the implosion has significant 2D and/or 3D structure. While our implosions appear fairly round, and quasi-orthogonal MMI views at similar times yield similar results [e.g., shot 78197, blue and orange curves in fig. 4(b)], small-scale non-uniformities are clearly visible [e.g., fig. 2(b)]. We assessed the effect of non-uniformities for shot 78199 (TIM 3, frame 2) by dividing the narrowband images of fig. 2(b) into three wedges of $120^{\circ}$ each and doing independent analysis on each wedge to obtain $n_{e}$ and $T_{e}$ profiles; the standard deviations of $n_{e}(r)$ and $T_{e}(r)$ across the three wedges are similar to the error bars already considered (as shown in fig. 3) and thus would not substantially increase the final error bars shown in fig. 4. Future work should employ both 2D 31 and 3D 43. reconstruction techniques to further assess both $2 \mathrm{D}$ and 3D effects on our analysis. Secondly, unaccounted-for $\mathrm{CH}$ shell mix into the hot spot affects the species balance in the quasi-neutrality condition used in our analysis by causing overestimates of the inferred $n_{D}$, which affects the inferred $f_{A r}$. However, we have estimated that the local $\mathrm{CH}$ fraction in the hot spot must exceed an unrealistically large value $\sim 10 \%$ before it could affect our conclusion of D/Ar separation presented in figs. 4(a) and 4(b). Finally,
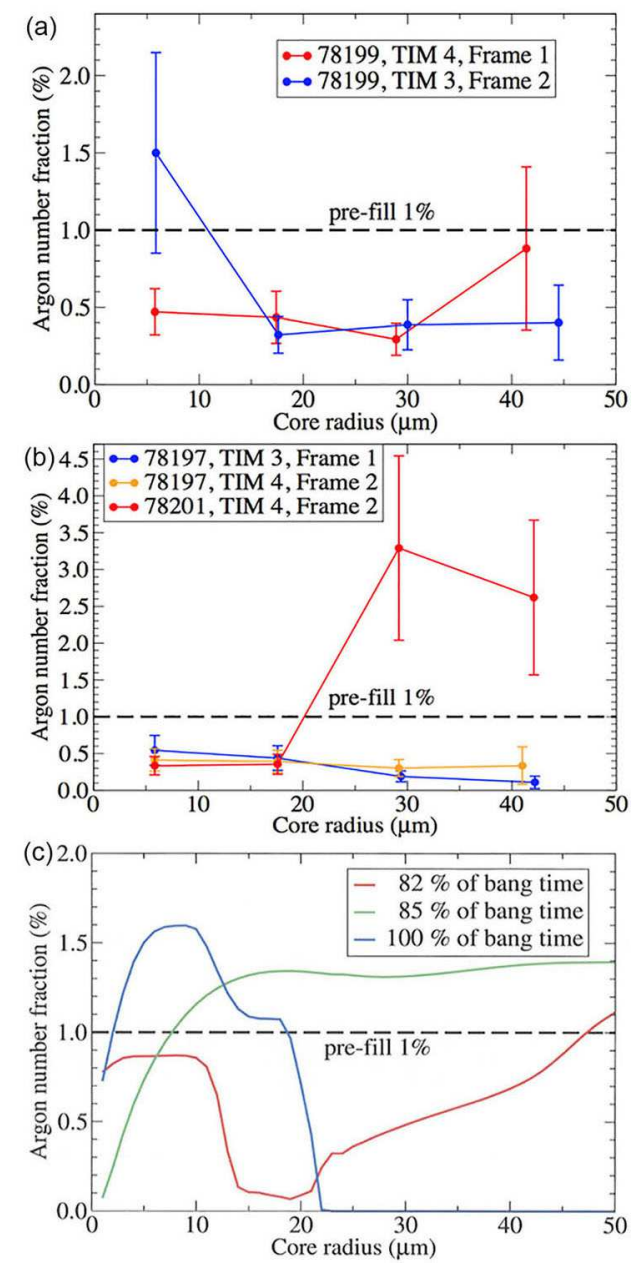

Fig. 4: Argon number fraction $f_{A r}$ versus $r$ for (a) shot 78199 $($ red $\approx 1.10 \mathrm{~ns}=84 \%$ of BT, blue $\approx 1.14 \mathrm{~ns}=87 \%$ of BT $)$, (b) shots 78197 and 78201 (blue $\approx 1.08 \mathrm{~ns}=82 \%$ of BT, orange $\approx 1.09 \mathrm{~ns}=83 \%$ of $\mathrm{BT}$, red $\approx 1.18 \mathrm{~ns}=87 \%$ of $\mathrm{BT})$, and (c) 1D post-shot xRAGE simulation (shot 78199) including a two-ion-species transport model [16].

our use of the known initial amount of Ar in the fuel region to infer absolute $n_{A r}$ from $n_{u}$ (arbitrary units) means that $n_{A r}$ is overestimated if Ar leaks into the shell. The latter could be a possible explanation for why $f_{A r}(r)<1 \%$ everywhere for shot 78197 [fig. 4(b)], keeping in mind that $f_{A r}(r)$ is extracted only from regions with visible, analyzable Ar spectral emission. Resolution of the shell/gas-mix issues requires further work, including the use of $N$-species ion-diffusion models that can model both the D/Ar separation and gas/shell mix.

Next, we interpret our results via a post-shot, 1D simulation of shot 78199 using xRAGE [17] with a two-ionspecies transport model [16]. Figure 4(c) shows $f_{A r}(r)$ for shot 78199 , revealing that $f_{A r}$ is reduced ahead of the incoming first shock and enhanced behind it [green and red curves of fig. 4(c)]. After shock reflection (at $\approx 1.18 \mathrm{~ns}$ and $\approx 87 \%$ of neutron bang time in the simulation), $f_{A r}$ is enhanced throughout much of the hot spot 
(up to $\approx 20 \mu \mathrm{m}$ ) through neutron bang time [blue curve of fig. 4(c)]. Furthermore, when ion thermo-diffusion, which is the strongest expected contributor to interspecies separation between $\mathrm{D}$ and $\mathrm{Ar}$, is turned off in the calculation, the $f_{A r}$ enhancement and depletion largely disappear. Independently, these effects are also seen in post-shot simulations from another radiation-hydrodynamics code, to which a multi-ion-species diffusion model has been added 44 and recently updated to include ion thermo-diffusion [15, 45,47. Additional detailed comparisons between our data and simulation results from both codes will be reported elsewhere. While detailed validation is beyond the scope of this work, note that there is reasonable agreement between the data and two key aspects of the postshot simulation of shot 78199: (1) the peak magnitude of $f_{A r}(\approx 1.5 \%)$, i.e., compare maxima of blue curves in figs. 4(a) and $4(\mathrm{c})$, and (2) the qualitative evolution of $f_{A r}$, in that $f_{A r}$ is below $1 \%$ nearer the origin at an earlier time [red curves of figs. 4(a) and 4(c)] and above 1\% nearer the origin at a later time [blue curves of figs. 4(a) and $4(\mathrm{c})]$. Differences in the details of the $f_{A r}$-profile evolution between the observations and xRAGE simulation could arise because (1) there could be more D enhancement ahead of the shock front than the xRAGE prediction due to kinetic effects near the shock front, and (2) shell mix, which affects $f_{A r}$ through an altered species balance in the quasi-neutrality condition, is not included in the xRAGE simulation.

Additional evidence supports our identification of interspecies ion separation. The FESTR code 41] is used to find the best spectral fit to radially resolved Ar spectral data. In all cases examined, when $f_{A r}$ is allowed to be a free parameter in the fitting instead of being fixed at the initial pre-fill value of $1 \%$, the fit is improved, e.g., $36 \%$ improvement in normalized $\chi^{2}$ for the third radial zone (corresponding to $r \approx 30 \mu \mathrm{m}$ ) of the data in fig. 2 ,

In conclusion, we have reported direct experimental evidence for interspecies ion separation in an ICF implosion, via detailed analyses of imaging x-ray-spectroscopy data. These direct-drive OMEGA implosions of plastic capsules with $\mathrm{D}_{2} / \mathrm{Ar}$ gas fill were designed to maximize interspecies ion thermo-diffusion between ion species of large mass and charge difference. This campaign benefitted from pre- and post-shot radiation-hydrodynamic simulations including a first-principles treatment of interspecies ion diffusion. The simulations show that $f_{A r}$ is enhanced and depleted, respectively, in front of and behind the first incoming shock, with $f_{A r}$ enhancement persisting at the center from firstshock convergence through neutron bang time. Our data agree reasonably well with the calculated effects on $f_{A r}(r)$ due to the incoming and rebounding first shock, but more detailed validation, which is beyond the scope of this work, is needed. The experimental Ar K-shell spectral signatures are overtaken by bremsstrahlung continuum by the time of neutron bang time (around the time of peak compression), and thus we are unable to report whether interspecies separation is observed through bang time. Nevertheless, this first result using x-ray spectroscopy to detect interspecies ion separation encourages further work toward establishing a validated capability to address its role in yield degradation of ignition-scale ICF implosions.

\section{$* * *$}

We acknowledge R. Aragonez, T. Archuleta, J. Cobble, J. Fooks, V. Glebov, M. Schoff, T. Sedillo, C. Sorce, R. Staerker, N. Whiting, B. Yaakobi, and the OMEGA operations team for their support in experimental planning, execution, and providing processed x-ray and neutron data. This work was supported by the LANL ICF and ASC (Advanced Simulation and Computing) Programs under U.S. Department of Energy contract no. DE-AC52-06NA25396.

\section{REFERENCES}

[1] Amendt P., Landen O. L., Robey H. F., Li C. K. and Petrasso R. D., Phys. Rev. Lett., 105 (2010) 115005.

[2] Amendt P., Wilks S. C., Bellei C., Li C. K. and Petrasso R. D., Phys. Plasmas, 18 (2011) 056308.

[3] Bellei C., Amendt P. A., Wilks S. C., Haines M. G., Casey D. T., Li C. K., Petrasso R. and Welch D. R., Phys. Plasmas, 20 (2013) 012701.

[4] Rygg J. R., Frenje J. A., Li C. K., Séguin F. H., Petrasso R. D., Delettrez J. A., Glebov V. Y., Goncharov V. N., Meyerhofer D. D., Regan S. P., Sangster T. C. and Stoeckl C., Phys. Plasmas, 13 (2006) 052702.

[5] Wilson D. C., Kyrala G. A., Benage J. F., Wysocki F. J., Gunderson M. A., Garbett W. J., V. Yu. Glebov, Frenje J., Yaakobi B., Herrmann H. W., Cooley J. H., Welser-Sherrill L., Horsfield C. J. and Roberts S. A., J. Phys. Conf. Ser., 112 (2008) 022015.

[6] Dodd E. S., Benage J. F., Kyrala G. A., Wilson D. C., Wysocki F. J., Seka W., Glebov V. Y., Stoeckl C. and Frenje J. A., Phys. Plasmas, 19 (2012) 042703.

[7] Herrmann H. W., Langenbrunner J. R., Mack J. M., Cooley J. H., Wilson D. C., Evans S. C., Sedillo T. J., Kyrala G. A., Caldwell S. E., Young C. S., Nobile A., Wermer J., Paglieri S., McEvoy A. M., Kim Y., Batha S. H., Horsfield C. J., Drew D., Garbett W., Rubery M., Glebov V. Y., Roberts S. and Frenje J. A., Phys. Plasmas, 16 (2009) 056312.

[8] Casey D. T., Frenje J. A., Gatu Johnson M., Manuel M. J.-E., Rinderknecht H. G., Sinenian N., Séguin F. H., Li C. K., Petrasso R. D., Radha P. B., Delettrez J. A., Glebov V. Y., Meyerhofer D. D., Sangster T. C., McNabb D. P., Amendt P. A., Boyd R. N., RygG J. R., Herrmann H. W., Kim Y. H. and Bacher A. D., Phys. Rev. Lett., 108 (2012) 075002.

[9] Rinderknecht H. G., Sio H., Li C. K., Hoffman N., Zylstra A. B., Rosenberg M. J., Frenje J. A., Gatu Johnson M., Séguin F. H., Petrasso R. D., Betti R., Yu Glebov V., Meyerhofer D. D., Sangster T. C., Seka W., Stoeckl C., Kagan G., Molvig K., Bellei C., Amendt P., Landen O., RygG J. R., 
Smalyuk V. A., Wilks S., Greenwood A. and Nikroo A., Phys. Plasmas, 21 (2014) 056311.

[10] Rosenberg M. J., Séguin F. H., Amendt P. A., Atzeni S., Rinderknecht H. G., Hoffman N. M., Zylstra A. B., Li C. K., Sio H., Gatu Johnson M., Frenje J. A., Petrasso R. D., Glebov V. Y., Stoeckl C., Seka W., Marshall F. J., Delettrez J. A., Sangster T. C., Betti R., Wilks S. C., Pino J., Kagan G., Molvig K. and Nikroo A., Phys. Plasmas, 22 (2015) 062702.

[11] Rinderknecht H. G., Rosenberg M. J., Li C. K., Hoffman N. M., Kagan G., Zylstra A. B., Sio H., Frenje J. A., Gatu Johnson M., Séguin F. H., Petrasso R. D., Amendt P., Bellei C., Wilks S., Delettrez J., Glebov V. Y., Stoeckl C., Sangster T. C., Meyerhofer D. D. and Nikroo A., Phys. Rev. Lett., 114 (2015) 025001.

[12] Amendt P., Bellei C. and Wilks S., Phys. Rev. Lett., 109 (2012) 075002.

[13] Kagan G. and Tang X. Z., Phys. Rev. Lett., 109 (2012) 269501.

[14] Kagan G. and Tang X.-Z., Phys. Plasmas, 19 (2012) 082709 .

[15] Kagan G. and Tang X.-Z., Phys. Lett. A, 378 (2014) 1531 .

[16] Molvig K., Simakov A. N. and Vold E. L., Phys. Plasmas, 21 (2014) 092709.

[17] Gittings M., Weaver R., Clover M., Betlach T., Byrne N., Coker R., Dendy E., Hueckstaedt R., New K., Oakes W. R., Ranta D. and Stefan R., Comput. Sci. Discover, 1 (2008) 015005.

[18] Vold E. L., Joglekar A. S., Ortega M. I., Moll R., Fenn D. and Molvig K., Phys. Plasmas, 22 (2015) 112708.

[19] Larroche O., Phys. Plasmas, 19 (2012) 122706.

[20] Inglebert A., Canaud B. and Larroche O., Europhys. Lett., 107 (2014) 65003.

[21] Bellei C., Rinderknecht H., Zylstra A., RosenBerg M., Sio H., Li C. K., Petrasso R., Wilks S. C. and Amendt P. A., Phys. Plasmas, 21 (2014) 056310.

[22] Kwan T., Le A., Schmitt M., Herrmann H. and Batha S., Bull. Amer. Phys. Soc., 60 (2015) 324.

[23] Edwards M. J., Patel P. K., Lindl J. D., Atherton L. J., Glenzer S. H., HaAn S. W., Kilkenny J. D., Landen O. L., Moses E. I., Nikroo A., Petrasso R., Sangster T. C., Springer P. T., Batha S., Benedetti R., Bernstein L., Betti R., Bleuel D. L., Boehly T. R., Bradley D. K., Caggiano J. A., Callahan D. A., Celliers P. M., Ceruan C. J., Chen K. C., Clark D. S., Collins G. W., Dewald E. L., Divol L., Dixit S., Doeppner T., Edgell D. H., Fair J. E., Farrell M., Fortner R. J., Frenje J., Gatu Johnson M. G., Giraldez E., Glebov V. Y., Grim G., Hammel B. A., Hamza A. V., Harding D. R., Hatchett S. P., Hein N., Herrmann H. W., Hicks D., Hinkel D. E., Hoppe M., Hsing W. W., Izumi N., Jacoby B., Jones O. S., Kalantar D., Kauffman R., Kline J. L., Knauer J. P., Koch J. A., Kozioziemski B. J., Kyrala G., LaFortune K. N., Pape S. L., Leeper R. J., Lerche R., Ma T., MacGowan B. J., MacKinnon A. J., Macphee A., Mapoles E. R., Marinak M. M., Mauldin M., McK- enty P. W., Meezan M., Michel P. A., Milovich J., Moody J. D., Moran M., Munro D. H., Olson C. L., Opachich K., Pak A. E., Parham T., Park H.-S., Ralph J. E., Regan S. P., Remington B., Rinderknecht H., Robey H. F., Rosen M., Ross S., Salmonson J. D., Sater J., Schneider D. H., Sguin F. H., Sepke S. M., Shaughnessy D. A., Smalyuk V. A., Spears B. K., Stoeckl C., Stoeffl W., Suter L., Thomas C. A., Tommasini R., Town R. P., WeBer S. V., Wegner P. J., Widman K., Wilke M., Wilson D. C., Yeamans C. B. and Zylstra A., Phys. Plasmas, 20 (2013) 070501.

[24] Boehly T. R., Brown D. L., Craxton R. S., Keck R. L., Knauer J. P., Kelly J. H., Kessler T. J., Kumpan S. A., Loucks S. J., Letzring S. A., Marshall F. J., McCrory R. L., Morse S. F. B., Seka W., Soures J. M. and Verdon C. P., Opt. Commun., 133 (1997) 495.

[25] Haynes D. A., Garber D. T., Hooper C. F., Mancini R. C., Lee Y. T., Bradley D. K., Delettrez J., Epstein R. and Jahnimagi P. A., Phys. Rev. E, 53 (1996) 1042.

[26] Golovkin I., Mancini R., Louis S., Ochi Y., Fujita K., Nishimura H., Shirga H., Miyanaga N., Azechi H., Butzbach R., Uschmann I., Förster E., Delettrez J., Koch J., Lee R. W. and Klein L., Phys. Rev. Lett., 88 (2002) 045002.

[27] Regan S. P., Delettrez J. A., Epstein R., JaAnimagi P. A., YaAkobi B., Smalyuk V. A., Marshall F. J., Meyerhofer D. D., Seka W., Haynes D. A., Golovkin I. E. and Hooper C. F., Phys. Plasmas, 9 (2002) 1357.

[28] Welser-Sherrill L., Mancini R. C., Koch J. A., Izumi N., Tommasini R., HaAn S. W., Haynes D. A., Golovkin I. E., MacFarlane J. J., Delettrez J. A., Marshall F. J., Regan S. P., Smalyuk V. A. and Kyrala G., Phys. Rev. E, 76 (2007) 056403.

[29] Nagayama T., Mancini R. C., Florido R., Tommasini R., Koch J. A., Delettrez J. A., Regan S. P. and Smalyuk V. A., J. Appl. Phys., 109 (2011) 093303.

[30] Florido R., Mancini R. C., Nagayama T., Tommasini R., Delettrez J. A., Regan S. P. and Yaakobi B., Phys. Rev. E, 83 (2011) 066408.

[31] Nagayama T., Mancini R. C., Florido R., Mayes D., Tommasini R., Koch J. A., Delettrez J. A., Regan S. P. and Smalyuk V. A., Phys. Plasmas, 21 (2014) 050702 .

[32] Marinak M. M., Kerbel G. D., Gentile N. A., Jones O., Munro D., Pollaine S., Dittrich T. R. and HaAn S. W., Phys. Plasmas, 8 (2001) 2275.

[33] Koch J. A., Barbee T. W., Izumi N., Tommasini R., Mancini R. C., Welser L. A. and Marshall F. J., Rev. Sci. Instrum., 76 (2005) 073708.

[34] Nagayama T., Mancini R. C., Mayes D., Tommasini R. and Florido R., Rev. Sci. Instrum., 86 (2015) 113505.

[35] The MMI data is corrected as follows. First, the streaked $\mathrm{x}$-ray-spectroscopy data (SSCA) is corrected by matching the time-integrated SSCA data to the space- and timeintegrated, absolutely calibrated x-ray-spectroscopy data (XRS2). Then the space-integrated MMI spectrum is corrected to match the corrected SSCA data at the time of 
the MMI data.

[36] Sampson D. H., Zhang H. L. and Fontes C. J., Phys. Rep., 477 (2009) 111.

[37] Fontes C. J., Zhang H. L., J. Abdallah, Jr., Clark R. E. H., Kilcrease D. P., Colgan J., Cunningham R. T., Hakel P., Magee N. H. and Sherrill M. E., J. Phys. B, 48 (2015) 144014.

[38] J. Abdallah, Jr. and R. E. H. Clark, "Rate Equations for Plasmas in Non Local Thermodynamic Equilibrium," Los Alamos Technical Report LA-11926 (1990).

[39] C. J. Fontes, J. Colgan, and J. Abdallah, Jr., in Modern Methods in Collisional-Radiative Modeling of Plasmas, ed. Yu. Ralchenko (Springer, Switzerland, 2016), pp. 17-50.

[40] Hakel P., Kyrala G. A., Bradley P. A., KrasheninNikova N. S., Murphy T. J., SchmitT M. J., Tregillis I. L., Kanzleieter R. J., Batha S. H., Fontes C. J., Sherrill M. E., Kilcrease D. P. and Regan S. P., Phys. Plasmas, 21 (2014) 063306.

[41] P. Hakel, "FESTR: Finite-Element Spectral Transfer of Radiation spectroscopic modeling and analysis code," Comp. Phys. Comm., accepted (2016).

[42] T. R. Joshi, Ph.D. Dissertation, University of NevadaReno, 2015; submitted for publication.

[43] Nagayama T., Mancini R. C., Florido R., Mayes D., Tommasini R., Koch J. A., Delettrez J. A., Regan S. P. and Smalyuk V. A., Phys. Plasmas, 19 (2012) 082705 .

[44] Hoffman N. M., Zimmerman G. B., Molvig K., Rinderknecht H. G., Rosenberg M. J., Albright B. J., Simakov A. N., Sio H., Zylstra A. B., Gatu Johnson M., Sguin F. H., Frenje J. A., Li C. K., Petrasso R. D., Higdon D. M., Srinivasan G., Glebov V. Y., Stoeckl C., Seka W. and SAngSter T. C., Phys. Plasmas, 22 (2015) 052707.

[45] Schunk R. W., Rev. Geophys., 15 (1977) 429.

[46] Zhdanov V. M., Transport Processeses in Multicomponent Plasma (Taylor \& Francis, New York) 2002.

[47] Paquette C., Pelletier C., Fontaine G. and Michaud G., Astrophys. J. Suppl. Ser., 61 (1986) 177. 